\title{
First physics with CMS
}

\section{Martijn Mulders* for the CMS collaboration}

CERN, European Organization for Nuclear Research, Geneva, Switzerland

E-mail: martijn.muldersecern.ch

The year 2008 marked the completion of the Large Hadron Collider (LHC) and the LHC detectors. The construction and commissioning of these scientific instruments, unprecedented in scale, complexity and precision, involved a number of challenging physics measurements and analyses, even before the first proton-proton collisions. This report highlights a selection of such physics measurements for and by the Cosmic Muon Solenoid (CMS) detector. These first physics analyses at CMS illustrate the depth of our understanding of the detector and demonstrate its readiness for data taking.

Physics at LHC 2008

29 September - October 4, 2008

Split, Croatia

\footnotetext{
* Speaker.
} 


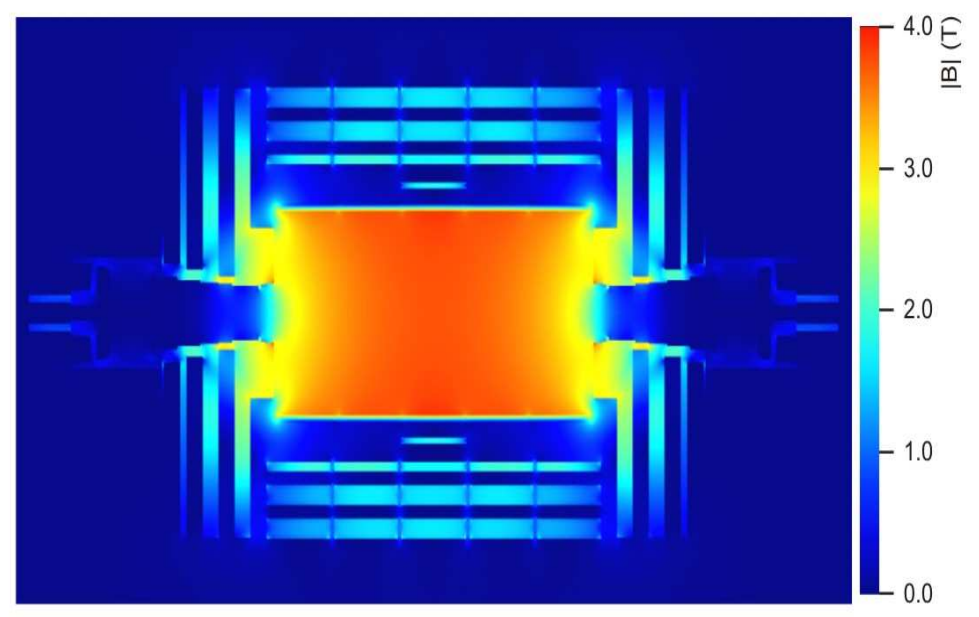

Figure 1: The calculated magnetic field strength $|\mathrm{B}|$ in CMS for a central field of $3.8 \mathrm{~T}$. The field is plotted for a cross-section of the CMS detector in the horizontal plane at the level of the beam line.

\section{Introduction}

The prospects of presenting first CMS results from LHC collisions in this report were shattered on the morning of September 19, 2008, when a serious incident in sector 3-4 of the LHC brought an end to an otherwise brilliant start-up phase that began 9 days earlier.

But even before collisions, the CMS detector is able to perform physics measurements, and physics measurements in turn are needed to finish commissioning the detector. An example of the latter category is the measurement and detailed analysis of the CMS magnetic field (section 叉), important for simulation, triggering and track reconstruction in CMS. Measurements with cosmic muons (section 3) and LHC beam induced muons (section $\$$ ) fall in both catagories. After these highlights of physics before collisions, section 5 briefly adresses plans for first physics after the first LHC collisions.

\section{Measurement of the CMS Magnetic Field}

The Compact Muon Solenoid (CMS) experiment [1] has been built as a general purpose detector with excellent tracking and particle identification capabilities [2] designed to fully explore the physics potential of the LHC[3]. Central in its design is a high-field (4T) super-conducting solenoid, with a $6 \mathrm{~m}$ diameter and a length of $12.5 \mathrm{~m}$.

A detailed map of the field in CMS (Figure 1) is provided for simulation, trigger, and track parameter reconstruction in CMS. This map is based on a three-dimensional model of the magnetic field in the whole CMS detector calculated using the TOSCA finite element program [7]. The model includes a description of the solenoid and the many iron volumes in the return yoke, reflected in the intricate structure of the field seen in Figure 1 . 

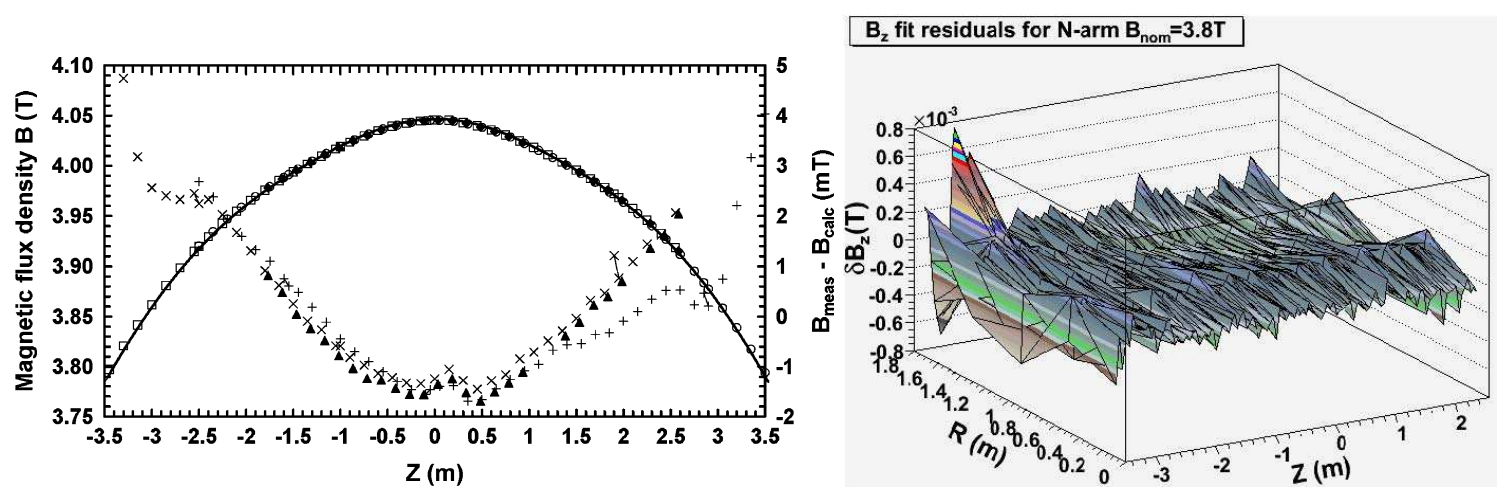

Figure 2: Left: Magnetic flux density (left scale) measured parallel to the coil axis at a radius of $1.724 \mathrm{~m}$ using an NMR probe, and two B-sensors located on the negative (open squares) and positive (open circles) fieldmapper arm. The smooth curve represents calculations done with the CMS TOSCA model. Triangles show the difference (right scale) between the NMR measurements and calculations, while the right and slanted crosses present the difference between B-sensors and the calculations. The model is normalized to get the measured $\mathrm{B}_{0}$ (on-axis) value of $4.0124 \mathrm{~T}$. The right plot shows the difference in $\mathrm{B}_{z}$ between the analytical field model and the measured values of the field, including post-calibration (see text), for the whole region inside the solenoid covered by one of the fieldmapper arms, at a field of $3.8 \mathrm{~T}$.

During the Magnet Test and Cosmic Challenge (MTCC) in the fall of 2006 [ 4 ], the CMS detector was closed for the first time, and the magnet was ramped to its design value of $B=4 \mathrm{~T}$. One of the goals of the MTCC was to perform accurate measurements of the magnetic field in CMS, at different settings of the field strength $(2.0,3.0,3.5,3.8$ and $4.0 \mathrm{~T})$. A field mapper was installed inside the solenoid, with the ability to perform precise measurements in a cylindrical volume with a radius of $1.724 \mathrm{~m}$ and a $7 \mathrm{~m}$ length [5].

The model agrees well with the field mapper results in the central region, as illustrated in Figure 2 (left plot). The agreement between calculations and measurements is better than $4 \mathrm{mT}$ in the whole range. The agreement between NMR and Hall probes is even better, suggesting that the measurements are more accurate than the TOSCA model.

A better fit to the measurements was obtained using a scalar potential approach. The scalar potential is formed as a linear combination of scalar basis functions (polynomials), where each basis function satisfies the Laplace equation and the gradient of the linear combination approximates well the field measurements. Using this analytical fit it was possible to post-calibrate the B-sensors to a level of $5 \cdot 10^{-5}$ at $4 \mathrm{~T}$. Comparing the result of the fit with the measurements of $B_{z}$ at each point $(r, z)$ in the mapping volume, an agreement better than $0.2 \mathrm{mT}$ is found for the tracker region $(r<1.2 \mathrm{~m})$, as shown in Figure 2 (right plot). More details are given in Ref. [8].

For the more complex field outside the solenoid, the TOSCA model is the only option. Comparisons of the TOSCA field model with flux loop measurements [6], indicate possible differences between the model and the real field in the iron of the return yoke of the order of $10 \%$. In the fall of 2008 a campaign has started to compare the TOSCA field with the $B$ values estimated using cosmic muons recorded in the CMS detector with the $B$ field turned on. Depending on the outcome of these measurements, the magnetic field map may have to be adapted using effective corrections obtained from in-situ measurements with muon tracks. 

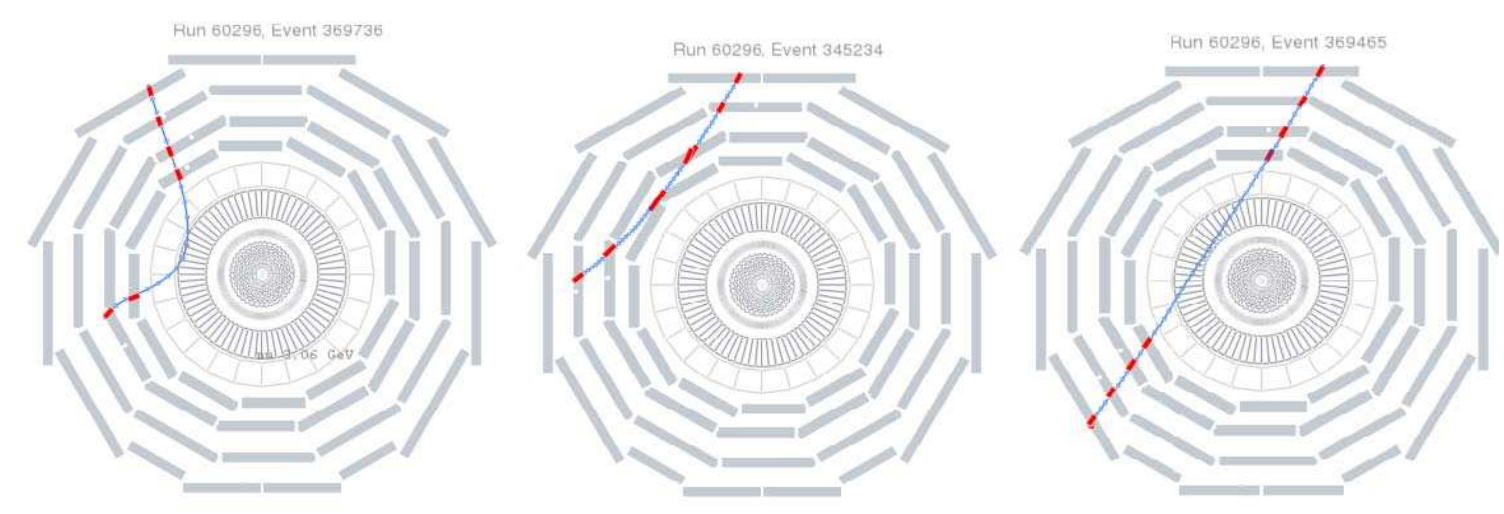

Figure 3: Three cosmic muon trajectories fitted with a dedicated muon tracking algorithm, for a 3T magnetic field. The reconstructed transverse momentum at closest approach to the beampipe is $3.1,6.2$ and $86 \mathrm{GeV} / c$ respectively.

\section{First CMS Physics Measurement with Cosmic Muons}

During the MTCC, a slice of the detector including a fraction of all subdetectors was integrated including the hardware, trigger, DAQ and offline analysis. More than 200 million cosmic muon events were recorded, with B field settings ranging from 0 to 4 Tesla.

This made it possible to measure the ratio of positively and negatively charged cosmic muons [0]. For the reconstruction of cosmics muons not pointing at the interaction region and crossing the whole detector, a dedicated muon reconstruction algorithm was used [10]. A few recent examples of muon tracks fitted with this dedicated algorithm are shown in Figure 3, recorded in 2008 during the first global run with a $3 \mathrm{~T}$ field.

To ensure charge symmetry in the acceptance, only muons passing through a 'charge' symmetric fiducial volume of the detector were selected. Monte Carlo simulation was used to correct for the effect of charge confusion due to detector resolution, and to correct the measured momentum spectrum for the average momentum loss passing through the CMS detector before reaching the bottom muon chambers $(\approx 7 \mathrm{GeV} / c)$. Alignment corrections (from survey data and from the measurements of the internal alignment of superlayers within a chamber) were applied to the hit positions before track reconstruction. The main remaining systematic uncertainties are due to charge confusion and alignment. As shown in Figure $€$ (left) the alignment error dominates for momenta above $100 \mathrm{GeV} / c$. The measured ratio

$$
R=1.282 \pm 0.004 \text { (statistical) } \pm 0.007 \text { (systematic) }
$$

agrees well with previous measurements of the most precise experiments, shown in Figure母(right).

The cosmics data also allow to test other aspects of muon reconstruction and identification. As an example in Figure 5 results are shown from one of the first global runs in 2008 including the silicon tracker (with $B=0 \mathrm{~T}$ ). This allowed testing of the high level trigger algorithms, matching 

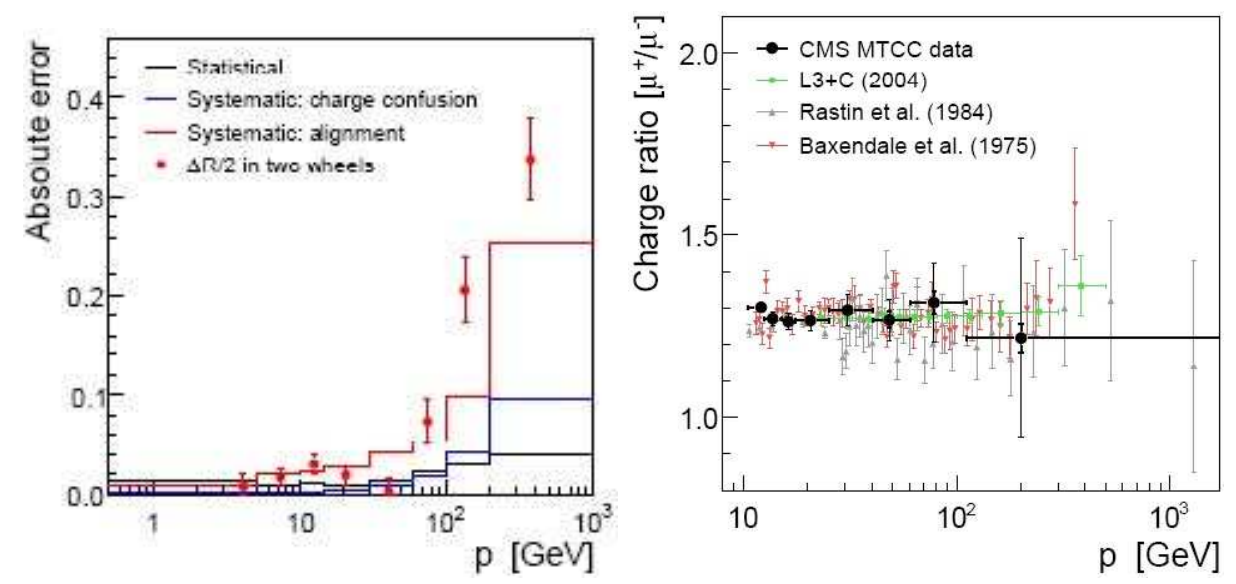

Figure 4: Main sources of systematic uncertainty for the muon charge ratio measurement as a function of momentum (left), and the CMS result compared to existing measurements (right).
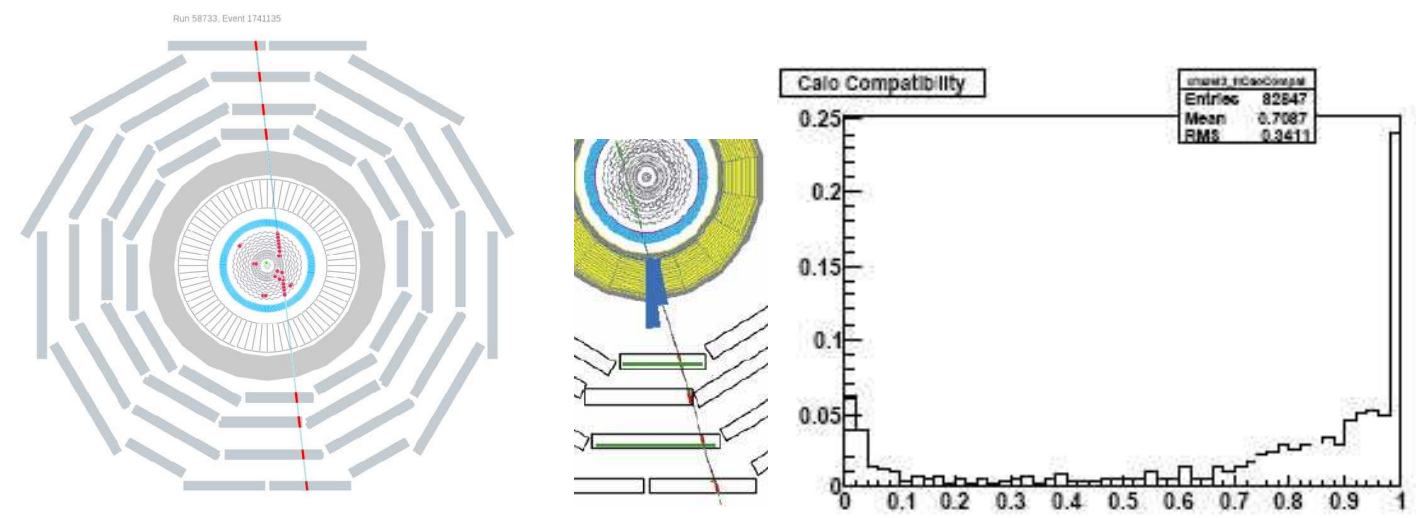

Figure 5: Example of a cosmic muon reconstructed as a di-muon event by the high-level trigger (left), a cosmic track matching an energy deposit in the hadronic calorimeter (middle), and a distribution of the muon compatibility (right), based on calorimeter deposits only.

of muon tracks to ECAL clusters and energy deposits in the HCAL, and the muon identification algorithm which determines a muon compatibility (between 0 and 1), based on the compatibility of matched calorimeter deposits with a muon hypothesis. Even though the algorithm was designed and tuned for pp collisions, the majority of cosmic muons is correctly assigned a high muon compatibility.

A small fraction $(0.005 \%)$ of the cosmic events are multi-muon events with more than 100 reconstructed muon segments. A muon segment can be reconstructed in each of the 4 layers of muon barrel stations, leading to a typical maximum of 8 segments per muon. With the magnetic field switched off, the precision with which the muon segments are parallel serves as an independent cross-check of the angular resolution of muon segment reconstruction. Conversely, the preliminary analysis of $2 \cdot 10^{3}$ multi-muon events showed that the origin of the showers had to be at least $0.5 \mathrm{~km}$ away from CMS, thus confirming their atmospheric origin. An example of a multi-muon event with $\approx 50$ muons is shown in Figure 6 (middle plot). 

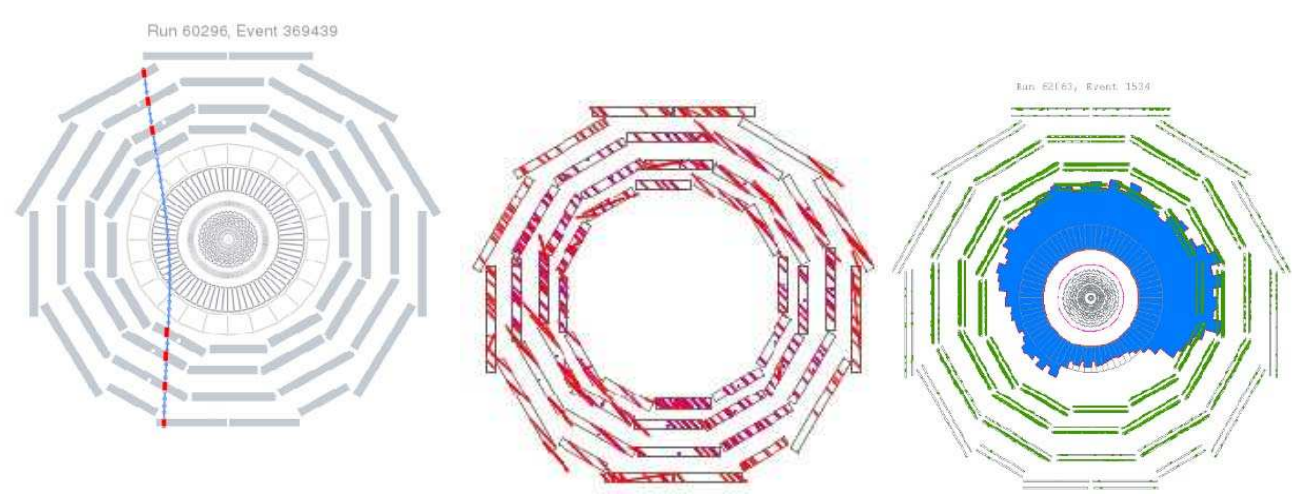

Figure 6: From left to right: a single cosmic event, a multi-muon cosmic event, and a beam shot event. These events, spanning a vast range of muon multiplicities $\left(1-10^{5}\right)$, were succesfully analyzed using full track reconstruction (left), local segments (middle) or reconstructed muon hits and energy deposits (right).

\section{Observation of the First Beam Induced Muons}

At start-up the LHC machine provided single shots of LHC beam 1 onto a collimator placed $150 \mathrm{~m}$ upstream of CMS. These shots were used to synchronize the CMS beam monitoring system to the beam timing. Using the beam monitoring system as Trigger, CMS recorded events in all sub-detectors except the silicon pixel and strip tracker which were left out of the run. In these shot events an estimated $10^{5}$ muons cross the volume of CMS, firing a large fraction of all channels in the central CMS detector. This allowed to time in the channels of several sub-detectors with a precision of about $1 \mathrm{~ns}$. Within hours after recording the first set of 17 beam shot events, the first analysis results were available, showing clear correlations between subdetectors in the energy recorded, as well as the angular distribution of the energy deposits. In some of the events the energy recorded in ECAL and HCAL exceeded $1000 \mathrm{TeV}$.

With a circulating LHC beam reaching more and more stable conditions, over a million beam halo muon events were recorded and reconstructed crossing the CMS detector from one Cathode Strip Chamber (CSC) endcap to the other. These events are used to study CSC chamber alignment. In Figure 7 the hit pattern of muon hits in the CSC chambers is shown, from a run dominated by cosmics (on the left), to a stable run with circulating beam where most of the hits are from beam halo muons, and the shape of the tunnel becomes clearly visible in the hit pattern.

\section{Prospects for First Physics with Collisions}

The measurements described in this report are only preparations for the real physics goals for which the CMS detector has been designed and built. Studies of the CMS physics analysis potential and plans for first physics analyses have been presented in detail in other reports at this conference. A comprehensive overview can be found in the CMS Physics TDR [2]. More recent physics analysis summaries are available on the CMS physics web page [11]. 

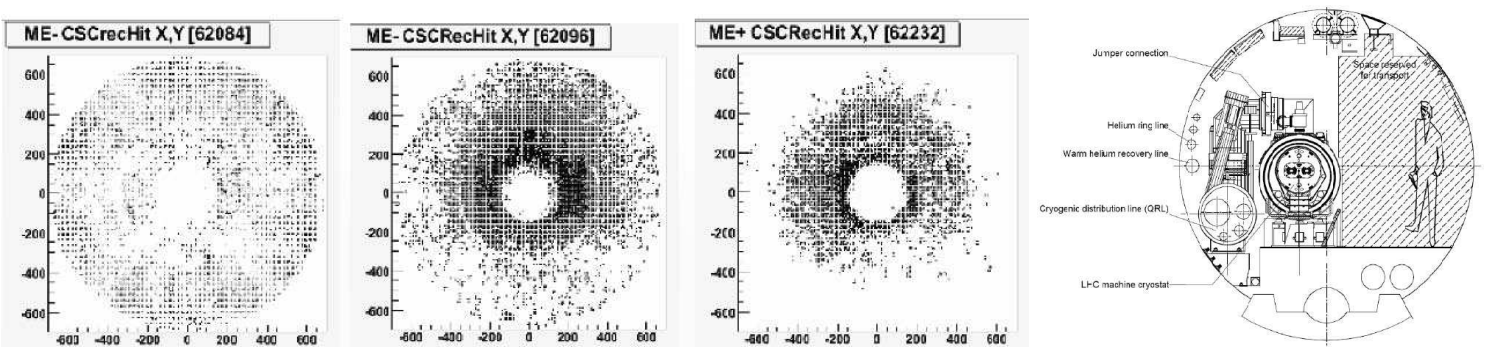

Figure 7: The pattern of hits in the endcap muon detectors, for a run with 50 passes of the beam (left), 100 s of passes (middle) and circulating beam (right). The emerging pattern resembles the shape of the LHC tunnel (far right).

\section{Conclusion}

After almost 20 years of design and construction, CMS has started taking data with cosmic muons and (single) LHC beams and recorded its first beam events. At these occasions the CMS detector and collaboration succeeded to quickly perform varied and meaningful analysis of a wide range of events, from single muon events to events with $10^{5}$ muons crossing the detector (see Figure 6). CMS is ready for first physics with LHC collisions in 2009.

\section{References}

[1] The CMS Collaboration, S Chatrchyan et al, The CMS experiment at the CERN LHC, 2008 JINST 3 S08004

[2] The CMS Collaboration, CMS Physics Technical Design Report, Volume 1: Detector Performance and Software, CERN/LHCC 2006-001;

The CMS Collaboration, CMS Physics Technical Design Report, Volume 2: Physics Performance, J. Phys. G: Nucl. Part. Phys., 34 (2007) 995.

[3] Lyndon Evans and Philip Bryant (editors), LHC Machine, 2008 JINST 3 S08001

[4] The CMS Collaboration, The CMS Magnet Test and Cosmic Challenge (MTCC Phase I and II), Operational Experience and Lessons Learnt, CMS NOTE 2007/005.

[5] V I Klyukhin et al, Measurement of the CMS Magnetic Field, IEEE Transactions on Applied Superconductivity, vol.18, no.2, June 2008

[6] V I Klyukhin et al, Measuring the Magnetic Field Inside the CMS Steel Yoke Elements, submitted to Transactions on Nuclear Science, proceedings of the IEEE NSS 2008 conference

[7] TOSCA/OPERA-3d Software, Vector Fields Ltd., Oxford, U.K.

[8] Vassili Maroussov, An Analytic Fit of the Measured Magnetic Field of the CMS Detector, PhD Thesis, Purdue University (2008)

[9] M. Aldaya, P. Garcia-Abia, Measurement of the Charge Ratio of Cosmic Muons using CMS data, CMS NOTE 2008/0016

[10] C. Liu, N. Neumeister, Reconstruction of Cosmic and Beam-Halo Muons, CMS NOTE 2008/001

[11] CMS Physics Results, https://twiki.cern.ch/twiki/bin/view/CMS/PhysicsResults 